\title{
The Largest Degrees of Irreducible Characters of the Symmetric Group
}

\author{
By John McKay
}

\begin{abstract}
The largest irreducible degrees and the partitions associated with them are tabulated for the symmetric group $\Sigma_{n}$ for $n$ up to 75. Analytic upper and lower bounds are derived for the largest degree.
\end{abstract}

Introduction. A question has been raised by Bivins and others [2]-namely:

For what irreducible representations of the symmetric group $\Sigma_{n}$ does the degree attain its maximal value and how does this maximum behave for large $n$ ?

This was apparently motivated by the practical considerations of number overflow in the computer but the same question arises in connection with sorting [1].

Each irreducible representation is associated with a partition $a=\left(a_{1}, a_{2}, \ldots, a_{k}\right)$, $a_{1} \geqslant a_{2} \geqslant \cdots \geqslant a_{k}>0$, of $n$. (We shall use $a \in n$ to mean that $a$ is one of the $p_{n}$ partitions of $n$.) Its degree is given by $[6$, p. 61] :

$$
d_{a}=n ! \frac{\Pi_{i<j}\left(b_{i}-b_{j}\right)}{\Pi_{i}\left(b_{i} !\right)}
$$

where $b_{i}=a_{i}+k-i$. A combinatorial interpretation of $d_{a}$ is that it is the number of ways the votes for $k$ candidates can be counted one at a time such that the final total number of votes cast is $n$ and at all stages in the counting $n_{1} \geqslant n_{2} \geqslant \cdots \geqslant n_{k}$, where $n_{i}$ is the current number of votes for candidate $i(i=1, \ldots, k)$ with finally $n_{i}=a_{i}$ $(i=1, \ldots, k)$.

Computation of the Maximal Degree. The calculations were made at Edinburgh University on a 4K 12-bit word length PDP8 computer using a multi-length routine for expansile integer multiplication. The strategy is straightforward. For increasing $n$, partitions of $n$ are generated in natural order ( $n$ first and $1^{n}$ last) as described in [11]. If a partition, $a$, precedes or coincides with its conjugate then the degree $d_{a}$ is computed as in the procedure degree of [9] but exponent arithmetic is used retaining integers throughout and avoiding unnecessary overflow. A description of exponent arithmetic appears in [10] but this description is slightly different from that used in this application, and the algorithm given there is a little garbled.

Three arrays are declared, ex, $h f a c, l f a c[2: N]$, where $N$ is the largest integer occurring as a natural factor (here $N$ is at most 75); ex $[n]$ contains the exponent of $n$ in the result and for all $n \leqslant N, h f a c[n]$ contains the largest prime factor of $n$ and $l f a c[n]$ contains the other factor. After initialization, the expression is evaluated by modifying the exponents in ex. For example, to divide by $k$ !:

$$
\text { for } i:=2 \text { step } 1 \text { until } k \text { do } \operatorname{ex}[i]:=\operatorname{ex}[i]-1 \text {; }
$$

Received June 5, 1975; revised September 30, 1975.

AMS (MOS) subject classifications (1970). Primary 20-04, 20C30; Secondary 05A15.

Key words and phrases. Irreducible representation, symmetric group, largest degree. 


\section{Maximal Irreducible Degrees of the Symmetric Group}

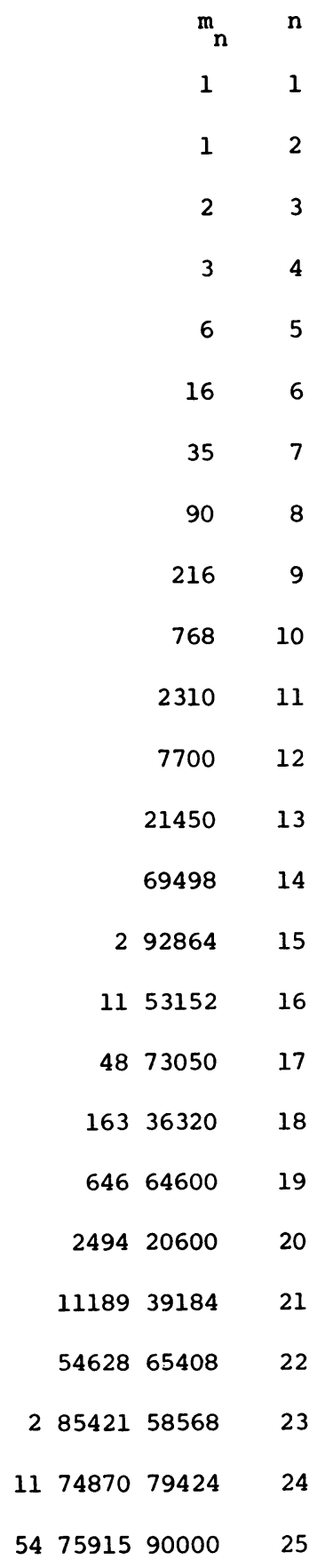

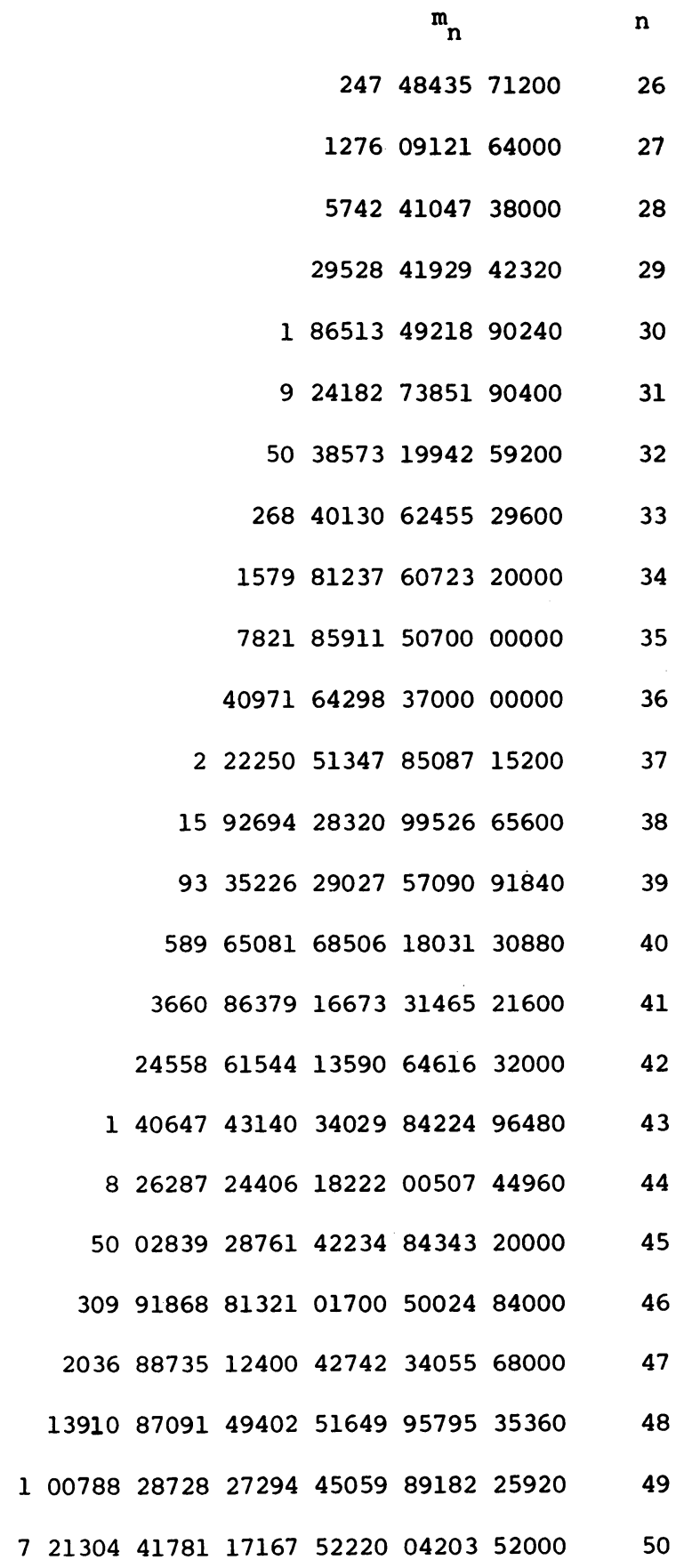




\begin{tabular}{|c|c|c|c|c|c|c|c|c|c|c|c|}
\hline & & & & \multicolumn{7}{|c|}{$m_{n}$} & $\mathrm{n}$ \\
\hline & & & & 54 & 86245 & 62826 & 89907 & 32913 & 48475 & 90400 & 51 \\
\hline & & & & 360 & 2717.3 & 44007 & 80906 & 66116 & 28632 & 57600 & 52 \\
\hline & & & & 2416 & 32801 & 79788 & 35907 & 70622 & 12235 & 61800 & $2=$ \\
\hline & & & & 16032 & 08919 & 82658 & 76501 & 24498 & 76481 & 40000 & 54 \\
\hline & & & 1 & 12332 & 94008 & 00148 & 07351 & 23185 & 00477 & 31500 & 55 \\
\hline & & & 7 & 80924 & 18237 & 44344 & 89607 & 49414 & 47168 & 50000 & 66 \\
\hline & & & 57 & 59492 & 68858 & 65309 & 68032 & 60594 & 83410 & 40000 & 1 \\
\hline & & & 392 & 04228 & 54325 & 17105 & 67342 & 81079 & 91024 & 00000 & 58 \\
\hline & & & 2843 & 60991 & 01639 & 97708 & 94957 & 04013 & 43897 & 60000 & 59 \\
\hline & & & 23219 & 99844 & 17184 & 55788 & 71179 & 66465 & 14524 & 16000 & 60 \\
\hline & & 1 & 98964 & 36084 & 33813 & 49744 & 27586 & 95268 & 29039 & 61600 & 1 \\
\hline & & 14 & 84932 & 70650 & 29909 & 32159 & 91941 & 84305 & 99280 & 64000 & 2 \\
\hline & & 112 & 80848 & 15471 & 49092 & 37752 & 38783 & 18899 & 58910 & 11200 & 3 \\
\hline & & 822 & 90818 & 64439 & 40221 & 23814 & 78702 & 63130 & 68681 & 13280 & 64 \\
\hline & & 6474 & 45118 & 59060 & 42071 & 22906 & 42354 & 58681 & 10615 & 19360 & 65 \\
\hline & & 49264 & 88872 & 06925 & 77842 & 72444 & 27860 & 67020 & 29690 & 57200 & 6 \\
\hline & 4 & 02557 & 12513 & 54748 & 85330 & 10840 & 14788 & 82368 & 98346 & 54000 & 57 \\
\hline & 30 & 47316 & 79121 & 25109 & 10697 & 47261 & 28840 & 64586 & 73715 & 20000 & 8 \\
\hline & 234 & 41791 & 16438 & 06987 & 94867 & 83935 & 00955 & 83550 & 21660 & 16000 & 69 \\
\hline & 1788 & 61125 & 56865 & 99443 & 44127 & 54238 & 97069 & 70842 & 13760 & 00000 & 70 \\
\hline & 14061 & 79814 & 66342 & 15100 & 92845 & 75298 & 46541 & 20312 & 24000 & 00000 & 1 \\
\hline 1 & 30752 & 27432 & 79523 & 21538 & 98976 & 09524 & 06388 & 52853 & 50440 & 96000 & 72 \\
\hline 10 & 99941 & 83391 & 42975 & 66548 & 10097 & 63063 & 04543 & 75434 & 51852 & 80000 & 73 \\
\hline 93 & 93814 & 29772 & 20073 & 46466 & 22546 & 26656 & 28282 & 24403 & 04999 & 04000 & \\
\hline & 91730 & & 1189 & 68765 & 20714 & 81759 & 17862 & 44539 & 84930 & 00000 & \\
\hline
\end{tabular}




\begin{tabular}{|c|c|c|c|c|}
\hline $\mathbf{n}$ & $\begin{array}{c}\sqrt{n !} \\
a\end{array}$ & $\begin{array}{c}=a \cdot 10^{b} \\
b\end{array}$ & $m_{n} / \sqrt{n !}$ & partition \\
\hline 1 & 1.00 & 0 & 1.000 & 1 \\
\hline 2 & 1.41 & 0 & 0.707 & 2 \\
\hline 3 & 2.45 & 0 & 0.816 & 2,1 \\
\hline 4 & 4.90 & 0 & 0.612 & 3,1 \\
\hline 5 & 1.10 & 1 & 0.548 & $3,1^{2}$ \\
\hline 6 & 2.68 & 1 & 0.596 & $3,2,1$ \\
\hline 7 & 7.10 & 1 & 0.493 & $4,2,1$ \\
\hline 8 & 2.01 & 2 & 0.448 & $4,2,1^{2}$ \\
\hline 9 & 6.02 & 2 & 0.359 & $4,3,1^{2}$ \\
\hline 10 & 1.90 & 3 & 0.403 & $4,3,2,1$ \\
\hline 11 & 6.32 & 3 & 0.366 & $5,3,2,1$ \\
\hline 12 & 2.19 & 4 & 0.352 & $5,3,2,1^{2}$ \\
\hline 13 & 7.89 & 4 & 0.272 & $5,4,2,1^{2}$ \\
\hline 14 & 2.95 & 5 & 0.235 & $6,4,2,1^{2}$ \\
\hline 15 & 1.14 & 6 & 0.256 & $5,4,3,2,1$ \\
\hline 16 & 4.57 & 6 & 0.252 & $6,4,3,2,1$ \\
\hline 17 & 1.89 & 7 & 0.258 & $6,4,3,2,1^{2}$ \\
\hline 18 & 8.00 & 7 & 0.204 & $7,4,3,2,1^{2}$ \\
\hline 19 & 3.49 & 8 & 0.185 & $7,5,3,2,1^{2}$ \\
\hline 20 & 1.56 & 9 & 0.160 & $7,5,3,2^{2}, 1$ \\
\hline 21 & 7.15 & 9 & 0.157 & $7,5,3,2^{2}, 1^{2}$ \\
\hline 22 & 3.35 & 10 & 0.163 & $7,5,4,3,2,1$ \\
\hline 23 & 1.61 & 11 & 0.178 & $7,5,4,3,2,1^{2}$ \\
\hline 24 & 7.88 & 11 & 0.149 & $8,5,4,3,2,1^{2}$ \\
\hline 25 & 3.9 .4 & 12 & 0.139 & $8,6,4,3,2,1^{2}$ \\
\hline
\end{tabular}




\begin{tabular}{|c|c|c|c|c|}
\hline $\mathbf{n}$ & $\begin{array}{c}\sqrt{n !} \\
a\end{array}$ & $\begin{array}{c}=a \cdot 10^{b} \\
b\end{array}$ & $m_{n} / \sqrt{n !}$ & partition \\
\hline 26 & 2.01 & 13 & 0.123 & $8,6,4,3,2,1^{3}$ \\
\hline 27 & 1.04 & 14 & 0.122 & $8,6,4,3,2^{2}, 1^{2}$ \\
\hline 28 & 5.52 & 14 & 0.104 & $8,6,5,3,2^{2}, 1^{2}$ \\
\hline 29 & 2.97 & 15 & 0.099 & $8,6,5,4,3,2,1$ \\
\hline 30 & 1.63 & 16 & 0.115 & $8,6,5,4,3,2,1^{2}$ \\
\hline 31 & 9.07 & 16 & 0.102 & $9,6,5,4,3,2,1^{2}$ \\
\hline 32 & 5.13 & 17 & 0.098 & $9,7,5,4,3,2,1^{2}$ \\
\hline 33 & 2.95 & 18 & 0.091 & $9,7,5,4,3,2,1^{3}$ \\
\hline 34 & 1.72 & 19 & 0.092 & $9,7,5,4,3,2^{2}, 1^{2}$ \\
\hline 35 & 1.02 & 20 & 0.077 & $9,7,6,4,3,2^{2}, 1^{2}$ \\
\hline 36 & 6.10 & 20 & 0.067 & $9,7,6,4,3^{2}, 2,1^{2}$ \\
\hline 37 & 3.71 & 21 & 0.060 & $10,8,6,4,3,2^{2}, 1^{2}$ \\
\hline 38 & 2.29 & 22 & 0.070 & $9,7,6,5,4,3,2,1^{2}$ \\
\hline 39 & 1.43 & 23 & 0.065 & $10,7,6,5,4,3,2,1^{2}$ \\
\hline 40 & 9.03 & 23 & 0.065 & $10,8,6,5,4,3,2,1^{2}$ \\
\hline 41 & 5.78 & 24 & 0.063 & $10,8,6,5,4,3,2,1^{3}$ \\
\hline 42 & 3.75 & 25 & 0.066 & $10,8,6,5,4,3,2^{2}, 1^{2}$ \\
\hline 43 & 2.46 & 26 & 0.057 & $11,8,6,5,4,3,2^{2}, 1^{2}$ \\
\hline 44 & 1.63 & 27 & 0.051 & $11,8,6,5,4,3,2^{2}, 1^{3}$ \\
\hline 45 & 1.09 & 28 & 0.046 & $11,9,7,5,4,3,2^{2}, 1^{2}$ \\
\hline 46 & 7.42 & 28 & 0.042 & $11,9,7,5,4,3,2^{2}, 1^{3}$ \\
\hline 47 & 5.09 & 29 & 0.040 & $10,8,7,6,5,4,3,2,1^{2}$ \\
\hline 48 & 3.52 & 30 & 0.039 & $11,8,7,6,5,4,3,2,1^{2}$ \\
\hline 49 & 2.47 & 31 & 0.041 & $11,9,7,6,5,4,3,2,1^{2}$ \\
\hline 5 & 1.74 & 32 & 0.041 & $11,9,7,6,5,4,3,2,1^{3}$ \\
\hline
\end{tabular}




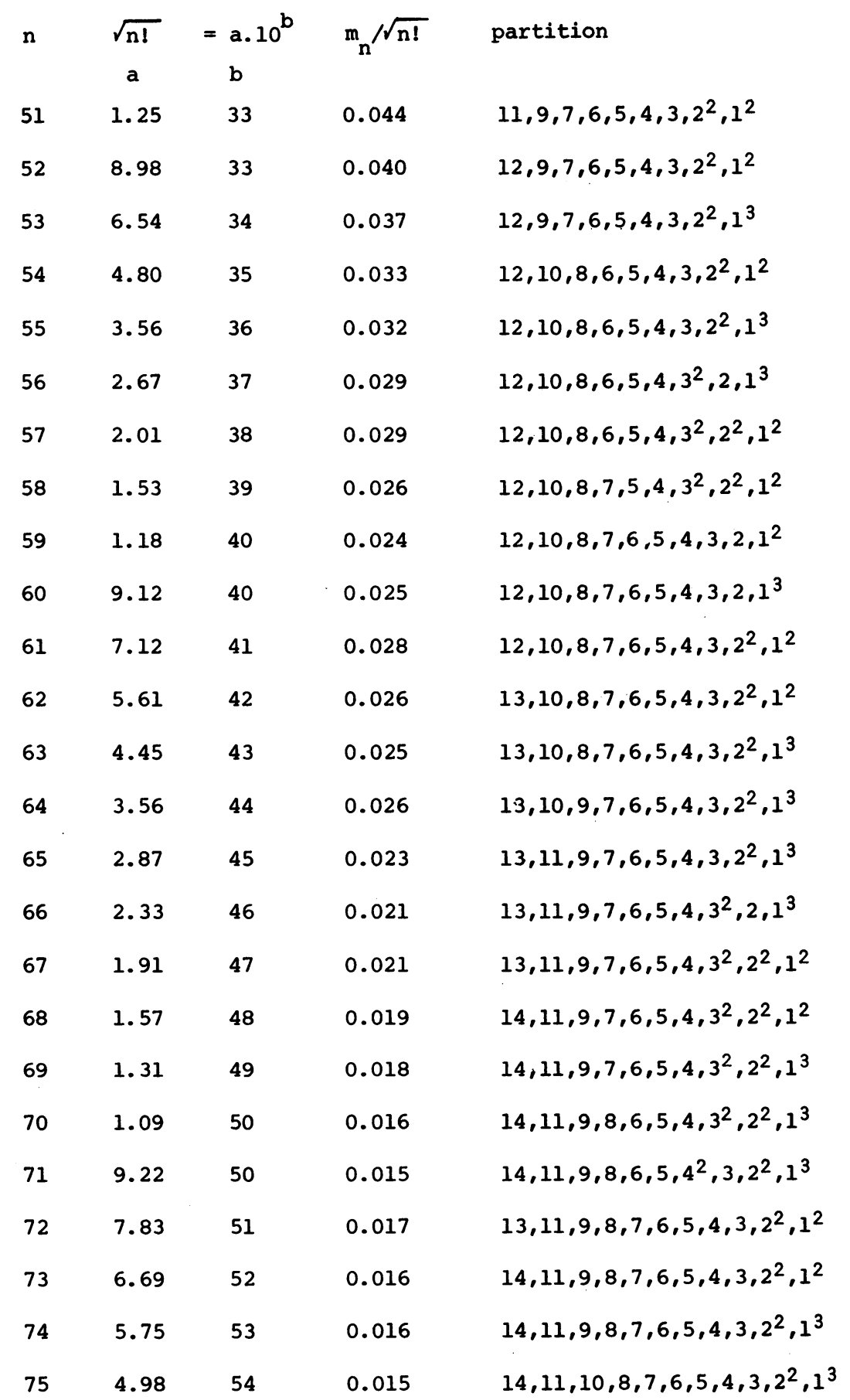


When complete, the result is reduced to a product of prime powers by decomposing the factors in decreasing order of magnitude into prime factors. The final numerical result may then be obtained. The computation of the cumulated product is speeded up by storing those prime powers that can be contained in a single-length word (viz. up to $\left.2^{12}-1\right)$. An ALGOL algorithm for the reduction is given by:

comment den is not needed if the result is known to be an integer, otherwise the result is given by num/den;

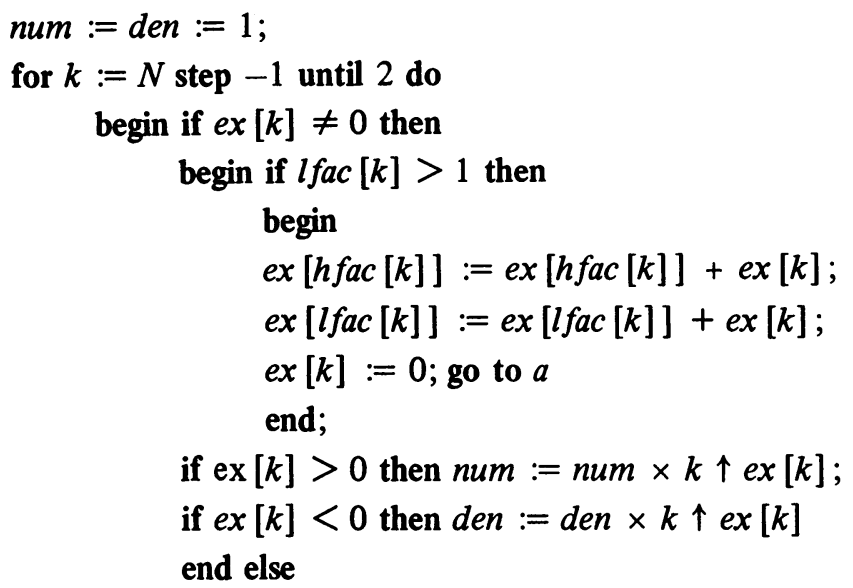

$a$ : end

The partial product num is stored as a multi-length integer and repeatedly multiplied by single-length integers to obtain the final value for the degree $d_{a}$. The machine used had no hardware multiplication and the worst case $\left(2^{12}-1 \times 2^{12}-1\right)$ single-length $x$ single-length multiplication took approximately $1 / 2$ millisec $=500$ instructions to complete.

The degrees were printed in decimal using Lunnon's [8] multi-length arithmetic package for Atlas.

The tables extend those of Comét [4] (up to $n=30$ ) and those of Baer and Brock [1] (up to $n=36$ ). They do not seem to reveal any simple recurrence between the partitions associated with the maximal degree for $\Sigma_{n}$ and those for $\Sigma_{k}(k<n)$. It is notable, however, that frequently a partition for the maximal degree for $\Sigma_{n}$ differs from that for the maximal degree for $\Sigma_{n-1}$ in a single part only.

Bounds for $\max d_{a}$. Upper and lower bounds for $m_{n}=\max _{a \in n} d_{a}$ are easy to find using group-theoretic facts concerning the characters of $\Sigma_{n}$. For a lower bound we have [5, p. 23] that the mean value of $d_{a}$ is given by $s_{n} / p_{n}$ where $s_{n}$ is the number of solutions to $x^{2}=1, x \in \Sigma_{n}$; viz.,

$$
s_{n}=\sum_{k=0}^{[1 / 2 n]} \frac{n !}{2^{k} k !(n-2 k) !} .
$$

The character column orthogonality relation on the degrees gives $n !=\Sigma_{a \in n} d_{a}^{2}$; hence, $s_{n} / p_{n} \leqslant m_{n} \leqslant(n !)^{1 / 2}$. 
Now Chowla, Herstein, and Moore [3] give the asymptotic $s_{n} \sim$ $2^{-1 / 2}(n / e)^{1 / 2 n} e^{n^{1 / 2}-1 / 4}$ and this, together with well-known approximations for $p_{n}$ and $n$ !, gives

$$
l_{n}=\frac{4 n \sqrt{3}}{e^{(\pi / 3) \sqrt{6 n}}} \cdot \frac{(n / e)^{1 / 2 n} e^{n^{1 / 2}}}{2^{1 / 2} e^{1 / 4}} \leqslant m_{n} \leqslant(2 \pi n)^{1 / 4}(n / e)^{1 / 2 n}=u_{n}
$$

from which

$$
\frac{u_{n}}{l_{n}}=\frac{k e^{t n^{1 / 2}}}{n^{3 / 4}}, \quad k=(\pi e / 288)^{1 / 4}, \quad t=\frac{\pi \sqrt{6}}{3}-1 .
$$

Remark. The referee has brought to my attention the work of Logan and Shepp [7] who have solved a continuous analogue of this problem and find their result not inconsistent with the partition tabulated here for $n=75$.

Added in Proof. It has been conjectured from the tables given here that $m_{n} \leqslant$ $\sqrt{n !} / n$, but Eric Regener has computed that the smallest value of $n$ for which the conjecture is false is $n=81$ which has a maximal partition of $15,12,10,9,7,6,5,4,3^{2}$, $2^{2}, 1^{3}$.

Department of Computer Science

Concordia University

1455 Maisonneuve West

Montreal, Quebec, Canada

1. R. M. BAER \& P. BROCK, "Natural sorting over permutation spaces," Math. Comp., v. 22, 1968, pp. 385-410. MR $37 \# 3800$.

2. R. L. BIVINS, N. METROPOLIS, P. R. STEIN \& M. B. WELLS, "Characters of the symmetric groups of degree 15 and 16," Math. Comp., v. 8, 1954, pp. 212-216. MR 16, 333.

3. S. CHOWLA, I. N. HERSTEIN \& W. K. MOORE, "On recursions connected with symmetric groups. I," Canad. J. Math., v. 3, 1951, pp. 328-334. MR 13, 10.

4. S. COMÉT, "Improved methods to calculate the characters of the symmetric group," Math. Comp., v. 14, 1960, pp. 104-117. MR 22 \#10212.

Mass., 1973.

5. W. FEIT, Characters of Finite Groups, Benjamin, New York, 1967. MR 36 \#2715.

6. D. E. KNUTH, The Art of Computer Programming. Vol. 3, Addison-Wesley, Reading,

7. B. F. LOGAN \& L. A. SHEPP, "A variational problem for random Young tableaux." (To appear.)

8. W. F. LUNNON, "Multi-length arithmetic for Atlas." (Unpublished.)

9. J. McKAY, "Symmetric group characters-Algorithm 307," Comm. ACM, v. 10, 1967, p. 451 ; ibid., v. 11,1968 , p. 14.

10. J. McKAY, "On the evaluation of multiplicative combinatorial expressions," Comm. $A C M$, v. 11, 1968, p. 392.

11. J. McKAY, "Partitions in natural order-Algorithm 371," Comm. ACM, v. 13, 1970, p. 52. 